\title{
Some Limiting Values and Two Error Estimation Procedures for Power Series Approximations
}

\section{By Moshe Mangad}

0. Abstract. Several limiting values concerned with the various remainder forms in the Taylor series expansion for one and two variables are developed. In addition, two error estimation procedures which are applicable to power series approximations of differential equations are also obtained.

1. Introduction. The integral remainder form in Taylor series expansion after the $n$th degree term of $f=f(x)$ in the neighborhood of $x=x_{0}$ is given [1] by

$$
R_{n}(x)=\int_{x_{0}}^{x} \frac{(x-t)^{n}}{n !} f^{(n+1)}(t) d t, \quad f^{(k)}(t) \equiv \frac{d^{k} f}{d t^{k}} .
$$

Rewriting Eq. (1) as

$$
R_{n}(x)=\frac{1}{n !} \int_{x_{0}}^{x}\left[(x-t)^{n-j} f^{(n+1)}(t)\right](x-t)^{j} d t, \quad j=0,1,2, \cdots, n,
$$

and using the mean value theorem for integrals, the following $(n+1)$ explicit remainders are obtained

$$
R_{n}(x)=\frac{h^{n+1}}{n !(j+1)}\left(1-\theta_{n}^{(j)}\right)^{n-j} f^{(n+1)}\left(x+\theta_{n}^{(j)}\right), \quad h \equiv x-x_{0},
$$

where $0<\theta_{n}{ }^{(j)}<1, j=0,1,2, \cdots, n$. The so-called Cauchy and Lagrange remainder forms are particular cases of (3) when $j=0$ and $j=n$, respectively.

2. The Limiting Values of $\theta_{n}{ }^{(j)}$. The aim in this section is to derive the limiting values of $\theta_{n}^{(j)}, j=0,1,2, \cdots, n$, as $h \rightarrow 0$ and an analogous one when $f$ is a function of two real variables, i.e., $f=f(x, y)$. An application of one of these limits to estimating a truncation error in the power series approximations of functions and solutions of differential equations is presented in Section 3, for sufficiently small mesh width $h$.

a. The one-variable case.

Theorem 1. If $f(x)$ and its $(n+1)$ derivatives are continuous on the interval $(a, b)$ and if $f^{(n+1)}\left(x_{0}\right) \neq 0, a<x_{0}<b$, then for $j=0,1,2, \cdots, n-1$,

$$
\lim _{h \rightarrow 0} \theta_{n}^{(j)}=1-\left(\frac{j+1}{n+1}\right)^{1 /(n-j)}
$$

Proof. Equating relation (3) with itself for $n=j$, then

$$
\frac{1}{(j+1) n !}\left(1-\theta_{n}{ }^{(j)}\right)^{n-j} f^{(n+1)}\left(x_{0}+\theta_{n}{ }^{(j)} h\right)=\frac{f^{(n+1)}\left(x_{0}+\theta_{n}{ }^{(n)} h\right)}{(n+1) !}
$$

Received August 26, 1966. Revised February 10, 1967. 
Letting $h \rightarrow 0$ on both sides of (5), one obtains $\left[1-\theta_{n}{ }^{(j)}\right]^{n-j} \rightarrow(j+1) /(n+1)$ as $h \rightarrow 0$, thus yielding relation (4).

Corresponding to $j=n$, the following result is valid.

Theorem 2. If $f(x)$ and its $(n+2)$ derivatives are continuous on the interval $(a, b)$ and if $f^{(n+2)}\left(x_{0}\right) \neq 0, a<x_{0}<b$, then

$$
\lim _{h \rightarrow 0} \theta_{n}^{(n)}=\frac{1}{n+2} .
$$

Proof. For $j=n$, we have that

$$
R_{n}(x)=\frac{h^{n+1}}{(n+1) !} f^{(n+1)}\left(x_{0}+\theta_{n}^{(n)} h\right), \quad 0<\theta_{n}^{(n)}<1, \quad h=x-x_{0} .
$$

Since $f^{(n+2)}(x)$ exists, one can then apply the mean value theorem to $f^{(n+1)}\left(x_{0}+\theta_{n}^{(n)} h\right)$, yielding

$$
f^{(n+1)}\left(x_{0}+\theta_{n}^{(n)} h\right)=f^{(n+1)}\left(x_{0}\right)+h \theta_{n}^{(n)} f^{(n+2)}\left(x_{0}+{\theta_{n}}^{(n)} \theta^{\prime} h\right),
$$

where $0<\theta_{n}^{(n)}, \theta^{\prime}<1$. Hence,

$$
R_{n}(x)=\frac{h^{n+1}}{(n+1) !} f^{(n+1)}\left(x_{0}\right)+\theta_{n}^{(n)} \frac{h^{n+2}}{(n+1) !} f^{(n+2)}\left(x_{0}+\theta_{n}^{(n)} \theta^{\prime} h\right) .
$$

Next, the continuity of $f^{(n+2)}\left(x_{0}\right)$ also implies that the Taylor series may have an additional term so that the new remainder is $R_{n+1}(x)$, where

$$
R_{n+1}(x)=\frac{h^{n+2}}{(n+2) !} f^{(n+2)}\left(x_{0}+\theta_{n+1}^{(n+1)} h\right), \quad 0<\theta_{n+1}^{(n+1)}<1 .
$$

Note that

$$
R_{n}(x)=\frac{h^{n+1}}{(n+1) !} f^{(n+1)}\left(x_{0}\right)+R_{n+1}(x) .
$$

Equating Eqs. (9) and (11) and using relation (10), it follows that

$$
\theta_{n}{ }^{(n)}=\frac{1}{n+2} \frac{f^{(n+2)}\left(x_{0}+\theta_{n+1}^{(n+1)} h\right)}{f^{(n+2)}\left(x_{0}+\theta_{n}^{(n)} \theta^{\prime} h\right)} \rightarrow \frac{1}{n+2}, \quad \text { as } \quad h \rightarrow 0 .
$$

This completes the proof of Theorem 2 .

b. The two-variables case. An identical result to (6) is obtained here for a function of two variables. The extension to higher-dimensional case will therefore be obvious.

Let us recall first that in Taylor series expansion of $f(x, y)\left\{\epsilon C^{n+1}(a, b ; c, d)\right\}$, the remainder after the $n$th partial derivative terms is given [1] by

$$
\begin{aligned}
R_{n}(x ; y)= & \frac{h^{n+1}}{(n+1) !} \frac{\partial^{n+1} f^{*}}{\partial x^{n+1}}\left(x_{0}+\theta_{n} h, y_{0}+\theta_{n} k\right)+\frac{h^{n}}{n !} \frac{k}{1 !} \frac{\partial^{n+1} f^{*}}{\partial x^{n} \partial y}+\cdots \\
& +\frac{k^{n+1}}{(n+1) !} \frac{\partial^{n+1} f^{*}}{\partial y^{n+1}}, \quad h \equiv x-x_{0}, \quad k \equiv y-y_{0} .
\end{aligned}
$$

Theorem 3. If $f(x, y)$ is of class $C^{n+2}(a, b ; c, d)$ and if all the $(n+2)$ partial 
derivatives are different from zero at some interior point $\left(x_{0}, y_{0}\right)$, then

$$
\lim _{(h, k) \rightarrow(0,0)} \theta_{n}=\frac{1}{n+2}, \quad h=x-x_{0}, \quad k=y-y_{0} .
$$

Proof. The proof follows from applying the mean value theorem to each factor in (13) and by considering $R_{n+1}(x ; y)$. The desired result will then follow from equating these two equivalent expressions and from taking the indicated limits. Indeed,

$$
\begin{aligned}
& \frac{\partial^{n+1} f^{*}}{\partial x^{n+1}}=\frac{\partial^{n+1} f\left(x_{0}, y_{0}\right)}{\partial x^{n+1}}+\theta_{n} h \frac{\partial^{n+2} f^{* *}}{\partial x^{n+2}}\left(x_{0}+\theta_{n} \theta^{\prime} h, y_{0}+\theta_{n} \theta^{\prime} k\right) \\
& +\theta_{n} k \frac{\partial^{n+2} f^{* *}}{\partial x^{n+1} \partial y} \\
& \frac{\partial^{n+1} f^{*}}{\partial y^{n} \partial y}=\frac{\partial^{n+1} f\left(x_{0}, y_{0}\right)}{\partial x^{n} \partial y}+\theta_{n} h \frac{\partial^{n+2} f^{* *}}{\partial x^{n+1} \partial y}+\theta_{n} k \frac{\partial^{n+2} f^{* *}}{\partial x^{n} \partial y^{2}} \\
& \frac{\partial^{n+1} f^{*}}{\partial y^{n+1}}=\frac{\partial^{n+1} f\left(x_{0}, y_{0}\right)}{\partial y^{n+1}}+\theta_{n} h \frac{\partial^{n+2} f^{* *}}{\partial x \partial y^{n+1}}+\theta_{n} k \frac{\partial^{n+2} f^{* *}}{\partial y^{n+2}} \\
& R_{n+1}(x ; y)=\frac{h^{n+2}}{(n+1) !} \frac{\partial^{n+2} f}{\partial x^{n+2}}\left(x_{0}+\theta_{n+1} h, y_{0}+\theta_{n+1} k\right) \\
& +\frac{h^{n+1}}{(n+1) !} \frac{k}{1 !} \frac{\partial^{n+2} f}{\partial x^{n+1} \partial y}+\cdots+\frac{k^{n+2}}{(n+2) !} \frac{\partial^{n+2} f}{\partial y^{n+2}} .
\end{aligned}
$$

Noting that

$$
\begin{aligned}
R_{n}(x ; y)= & R_{n+1}(x ; y)+\frac{h^{n+1}}{(n+1) !} \frac{\partial^{n+1} f\left(x_{0}, y_{0}\right)}{\partial x^{n+1}}+\frac{h^{n}}{n !} \frac{k}{1 !} \frac{\partial^{n+1} f\left(x_{0}, y_{0}\right)}{\partial x^{n} \partial y} \\
& +\cdots+\frac{k^{n+1}}{(n+1) !} \frac{\partial^{n+1} f\left(x_{0}, y_{0}\right)}{\partial y^{n+1}}
\end{aligned}
$$

and equating (13) with (17) using (16), the desired relation (14) follows at once.

Regarding functions of two and more real variables, one can generalize the result in Eq. (14) and assert that under the analogous hypotheses of Theorem 3 for $f=f\left(x_{1}, x_{2}, \cdots, x_{m}\right)$, we have

$$
\lim _{\left(h_{1}, h_{2}, \cdots, h_{m}\right) \rightarrow(0,0, \cdots, 0)} \theta_{n}=\frac{1}{n+2}, \quad h_{k}=x_{k}-x_{0 k}, \quad k=1,2, \cdots, m .
$$

3. Truncation Error Estimate with Application to Series Approximations. In this section the limiting value (6) will be utilized to obtain a truncation error estimate applicable to power series approximation of functions and approximate power series solution of differential equations. (The limiting values in (4) and (18) may be utilized analogously.) Assuming that $h \equiv x-x_{0}$ is sufficiently small, then substitution of the result in Eq. (6) into relation (7) leads to the following truncation error estimate $E_{n}\left(E_{n} \approx R_{n}\right)$ : 


$$
\begin{array}{r}
R_{n}=\frac{h^{n+1}}{(n+1) !} f^{(n+1)}\left(x_{0}+\theta_{n} h\right) \approx E_{n}=\frac{h^{n+1}}{(n+1) !} f^{(n+1)}\left(x_{0}+\frac{h}{n+2}\right) \\
n=0,1,2, \cdots .
\end{array}
$$

Note that expression (19) indicates that the error estimate is explicitly a function of the given $x_{0}$ and of the number of terms $n$ in the approximation.

Before applying estimate (19) note the following assertions. From Eq. (12) one has

$$
\theta_{n}=\frac{1}{n+2} \frac{f^{(n+2)}\left(x_{0}+\theta_{n+1} h\right)}{f^{(n+2)}\left(x_{0}+\theta_{n} \theta^{\prime} h\right)}=\frac{1}{n+2}+\epsilon_{n},
$$

where $\epsilon_{n}=\epsilon_{n}(h) \rightarrow 0$ for $h \rightarrow 0$. Applying next the mean value theorem to $f^{(n+1)}\left(x_{0}+\theta_{n} h\right)$, which may be rewritten as

$$
\begin{aligned}
f^{(n+1)}\left(x_{0}+\theta_{n} h\right) & =f^{(n+1)}\left[x_{0}+\frac{h}{n+2}+h\left(\theta_{n}-\frac{1}{n+2}\right)\right] \\
& =f^{(n+1)}\left(x_{0}+\frac{h}{n+2}+h \epsilon_{n}\right),
\end{aligned}
$$

there results

$$
\begin{aligned}
& f^{(n+1)}\left(x_{0}+\theta_{n} h\right)=f^{(n+1)}\left(x_{0}+\frac{h}{n+2}\right)+h \epsilon_{n} f^{(n+2)}\left(x_{0}+\frac{h}{n+2}+\theta h \epsilon_{n}\right), \\
& 0<\theta<1 .
\end{aligned}
$$

Therefore,

$$
R_{n}=\frac{h^{n+1}}{(n+1) !} f^{(n+1)}\left(x_{0}+\frac{h}{n+2}\right)+h^{n+2} \epsilon
$$

where

$$
\epsilon=\epsilon(h)=\frac{\epsilon_{n}}{(n+1) !} f^{(n+2)}\left(x_{0}+\frac{h}{n+2}+\theta h \epsilon_{n}\right) .
$$

If a Lipschitz condition is valid for $f^{(n+2)}(x)$, i.e.,

$$
\left|f^{(n+2)}\left(x_{1}\right)-f^{(n+2)}\left(x_{2}\right)\right| \leq L\left|x_{1}-x_{2}\right|, \quad a \leq x_{1}, x_{2} \leq b,
$$

it follows from Eq. (20) that

$$
\begin{aligned}
|\epsilon| & =\left|\frac{f^{(n+2)}\left(x_{0}+\frac{h}{n+2}+\theta h \epsilon_{n}\right)}{f^{(n+2)}\left(x_{0}+\theta_{n} \theta^{\prime} h\right)}\right| \frac{\left|f^{(n+2)}\left(x_{0}+\theta_{n+1} h\right)-f^{(n+2)}\left(x_{0}+\theta_{n} \theta^{\prime} h\right)\right|}{(n+2) !} \\
& \leq\left|1+\epsilon^{\prime}\right| L \frac{|h|}{(n+2) !},
\end{aligned}
$$

where $\epsilon^{\prime}=\epsilon^{\prime}(h) \rightarrow 0$ for $h \rightarrow 0$.

Hence, using the term $E_{n}$ in addition to the truncated Taylor series, one finds that the error involved in approximating $f(x)$ is of the order of magnitude 


$$
\left|h^{n+2} \epsilon(h)\right|=O\left(h^{n+3}\right) .
$$

a. Application to power series approximation of functions. The application of estimate (19) to power series approximation of functions will be considered here. Indeed, using the Taylor expansion for $f(x)$ together with estimate (19), one has

$$
\begin{aligned}
f(x) & =a_{0}+a_{1}\left(x-x_{0}\right)+a_{2}\left(x-x_{0}\right)^{2}+\cdots+a_{n}\left(x-x_{0}\right)^{n}+R_{n} \\
& \approx a_{0}+a_{1}\left(x-x_{0}\right)+a_{2}\left(x-x_{0}\right)^{2}+\cdots+a_{n}\left(x-x_{0}\right)^{n}+E_{n},
\end{aligned}
$$

where $a_{k}=f^{(k)}\left(x_{0}\right) / k !, k=0,1,2, \cdots, n ; a_{k}$ may also be obtained recursively via the Frobenius method of substitution. The incorporation of the estimate $E_{n}$, given in (19) of the truncation error into Eq. (21), improves the accuracy of the power series method in approximating functions by an $n$th degree polynomial. As for evaluating the expression $f^{(n+1)}\left(x_{0}+\left(x-x_{0}\right) /(n+2)\right)$ of $E_{n}$ it may be analytically obtained for example by $(n+1)$-successive differentiation of $f(x)$ evaluated at $x=x_{0}+\left(x-x_{0}\right) /(n+2)$. It may also be obtained approximately using linear extrapolation and interpolation, e.g.,

$$
f^{\prime \prime}\left(x_{0}+\frac{h}{3}\right)=\frac{1}{3}\left[4 f^{\prime \prime}(x)-f^{\prime \prime}(x-h)\right]+O\left(h^{2}\right), \quad h=x-x_{0} .
$$

Example 1. Let $f(x)=e^{x}$ and consider the Taylor series:

$$
f(x)=f(0)+x f^{\prime}(\theta x), \quad 0<\theta<1 .
$$

Then,

$$
e^{x}=1+x e^{\theta x}
$$

so that for $x=1, e=1+e^{\theta}$. $e^{\theta}$ here is the error when using one term of the Taylor series. It is found from tables that $\theta=.541 \cdots$. Hence, the true error is 1.71828 . On the other hand, the error estimate in our analysis is $e^{1 / 2}=1.6487$. The usual upper bound for the error is

$$
\max _{0 \leq \theta \leq 1} e^{\theta}=e=2.718 \cdots
$$

To see the improved accuracy in our method to approximate $e$, we incorporate the estimate of the truncation error into the approximate expression, thus yielding

$$
e \approx 1+e^{1 / 2}=2.6487 \ldots .
$$

If now we consider two terms of the Taylor series for approximating

$$
e \approx 1+1=2
$$

and if we consider three terms, we obtain

$$
e \approx 1+1+1 / 2=2.5 \text {. }
$$

It is seen, therefore, via this example that three terms in Taylor series do not yield the accuracy obtained by our method using the error estimate after one term. Only when four terms in Taylor series are taken to approximate $e$, i.e.,

$$
e=1+1+1 / 2+1 / 6=2.666 \cdots,
$$


do we obtain a more accurate result than the one suggested by our method of taking one term plus the error estimate. Our method, therefore, suggests that by incorporating the truncation error estimate $E_{n}$ into the approximation, there is less work involved in approximating functions, i.e., fewer number of terms need be accounted for.

b. Applications to power series solutions of differential equations. Consideration will be given here to the utilization of estimate (19) in obtaining approximate power series solutions to differential equations. The solution so obtained will be in a form of a truncated series. This truncated series is employed over a range that is limited by accuracy requirements. At the end of the interval a new series development is started, and so on (analytic continuation process).

Consider the system of first-order differential equations

$$
d x_{i} / d t \equiv \dot{x}_{i}=f_{i}\left(x_{1}, x_{2}, \cdots, x_{m}, t\right), \quad i=1,2, \cdots, m
$$

with the initial conditions

$$
x_{i}\left(t_{0}\right)=x_{i 0}, \quad i=1,2, \cdots, m .
$$

Assuming that $x_{1}(t), x_{2}(t), \cdots, x_{m}(t)$ are analytic and single-valued throughout some interval $\left(t_{0}, t_{1}\right)$, then in view of Eqs. (18)-(19), one may solve the system of equations (22) in a Taylor series as follows:

$$
\begin{aligned}
x_{i}(t)= & a_{0 i}+a_{1 i}\left(t-t_{0}\right)+a_{2 i}\left(t-t_{0}\right)^{2}+\cdots+a_{n i}\left(t-t_{0}\right)^{n} \\
& +\frac{\left(t-t_{0}\right)^{n+1}}{(n+1) !} \frac{d^{n+1} x_{i}}{d t^{n+1}}\left(t_{0}+\frac{t-t_{0}}{n+2}\right), \quad i=1,2, \cdots, m .
\end{aligned}
$$

The term

$$
\frac{d^{n+1} x_{i}}{d t^{n+1}}\left(t_{0}+\frac{t-t_{0}}{n+2}\right)
$$

may be obtained either analytically from the Taylor series to $n$th order found, say, by $n$-successive differentiations of $\dot{x}_{i}=f_{i}\left(x_{1}, \cdots, x_{m}, t\right)$ with respect to $t$ expanded about $t=t_{0}+\left|\left(t-t_{0}\right) /(n+2)\right|$ or numerically by use of linear extrapolation and interpolation (see Example 2 below). The constants $a_{j i}, j=0,1,2, \cdots, n, i=$ $1,2, \cdots, m$ may be either determined from the Taylor coefficient formula:

$$
a_{j i}=d^{j} x_{i}\left(t_{0}\right) / d t^{j}
$$

or from the Frobenius method of direct substitution. As in the case of approximating functions, the incorporation (as done in Eq. (22)) of the estimate of the truncation error (19) improves the accuracy of the power series method for solving differential equations.

Example 2. As a specific application of Eqs. (19)-(20), an algorithm will be developed below to obtain numerical solutions of a differential equation of the form $\ddot{x}=f(t, x)$.

Substituting $\theta_{1}=\frac{1}{3}$ in Taylor's formula for $n=1$, one obtains

$$
x(t+h)=x(t)+h \dot{x}(t)+h^{2} \ddot{x}(t+h / 3) / 2+h^{3} \epsilon(h) .
$$


If $\dddot{x}$ satisfies a Lipschitz condition, the error introduced at the step from $t$ to $t+h$ is $O\left(h^{4}\right)$. Linear extrapolation and interpolation yield

$$
\begin{aligned}
\ddot{x}(t+h / 3) & =(1 / 3)[4 \ddot{x}(t)-\ddot{x}(t-h)]+O\left(h^{2}\right), \\
\ddot{x}(t+2 h / 3) & =(1 / 3)[\ddot{x}(t)+2 \ddot{x}(t+h)]+O\left(h^{2}\right) .
\end{aligned}
$$

With

$$
x_{n}=x\left(t_{0}+n h\right), \quad \dot{x}_{n}=\dot{x}\left(t_{0}+n h\right), \quad f_{n}=f\left(t_{0}+n h, x_{n}\right),
$$

one has the following algorithm

$$
\begin{aligned}
& x_{n+1}=x_{n}+\dot{x}_{n} h+(1 / 6)\left[-f_{n-1}+4 f_{n}\right] h^{2}+O\left(h^{4}\right), \\
& x_{n+2}=x_{n}+2 \dot{x}_{n} h+(2 / 3)\left[f_{n}+2 f_{n+1}\right] h^{2}+O\left(h^{4}\right) \\
& \dot{x}_{n+2}=\dot{x}_{n}+(1 / 3)\left[f_{n}+4 f_{n+1}+f_{n+2}\right]+O\left(h^{5}\right)
\end{aligned}
$$

where the last recursion formula is simply Simpson's formula. We observe here that this two-step method promises a good accuracy in spite of its simplicity. However, the stability properties of the method need be further investigated.

4. An Alternate Truncation Error Estimate with Application to Power Series Solutions of Differential Equations. This section is concerned with deriving an alternate procedure for estimating truncation error in the power series approximation of differential equations. The procedure is independent of the results obtained in Sections 1 and 2 and it is presented here owing mainly to its applicability in estimating truncation errors. Unlike the method of Section 3 where high-order derivatives need be computed in obtaining the error estimate, the method proposed here is more direct as it does not involve such computations.

Consider again the system of differential equations (22) with the prescribed initial conditions (23). Assuming a power series solution for each $x_{i}(t)$ :

$$
x_{i}(t)=a_{0 i}+a_{1 i}\left(t-t_{0}\right)+\cdots+a_{n i}\left(t-t_{0}\right)^{n}+R_{n i} \equiv P_{n i}(t)+R_{n i},
$$
where

$$
P_{n i}(t) \equiv a_{0 i}+a_{1 i}\left(t-t_{0}\right)+\cdots+a_{n i}\left(t-t_{0}\right)^{n}
$$

is an $n$th degree polynomial in $t$ and where by means of Eq. (7)

$$
R_{n i}(t)=\frac{\left(t-t_{0}\right)^{n+1}}{(n+1) !} x_{i}^{(n+1)}\left(\xi_{i}\right), \quad t_{0}<\xi_{i}<t, \quad i=1,2, \cdots, m
$$

Next, we consider the Taylor series of one less order for the derivative, i.e.,

$$
\dot{x}_{i}(t)=\dot{P}_{n i}(t)+R_{n i}^{\prime}
$$

where

$$
R_{n i}^{\prime}=\frac{\left(t-t_{0}\right)^{n}}{n !} x_{i}^{(n+1)}\left(\xi_{i}{ }^{\prime}\right), \quad t_{0}<\xi_{i}{ }^{\prime}<t, \quad i=1,2, \cdots, m .
$$

If $t-t_{0}$ is small, the following approximations in terms of the mean values of $\xi_{i}, \xi_{i}{ }^{\prime}$ may be made: 


$$
\begin{aligned}
& x_{1}^{(n+1)}\left(\xi_{1}\right) \approx x_{1}^{(n+1)}\left(\xi_{1}{ }^{\prime}\right) \approx x_{1}{ }^{(m+1)}\left(\eta_{1}\right), \quad \text { say } \\
& \cdot \\
& \cdot \\
& \cdot \\
& x_{m}{ }^{(n+1)}\left(\xi_{m}\right) \approx x_{m}{ }^{(n+1)}\left(\xi_{m}{ }^{\prime}\right) \approx x_{m}{ }^{(n+1)}\left(\eta_{m}\right), \quad \text { say }
\end{aligned}
$$

where

$$
t_{0}<\eta_{1}, \eta_{2}, \cdots, \eta_{m}<t .
$$

With these assumptions one then has

$$
R_{n i}^{\prime}=\left((n+1) /\left(t-t_{0}\right)\right) R_{n i}, \quad i=1,2, \cdots, m .
$$

Substitutions into Eqs. (22) lead then to

$$
\dot{P}_{n i}(t)+\frac{n+1}{t-t_{0}} R_{n i}=f_{i}\left(t, P_{n 1}+R_{n 1}, P_{n 2}+R_{n 2}, \cdots, P_{n m}+R_{n m}\right) .
$$

The system of equations (32) constitute $m$ algebraic equations with the $m$ unknowns $R_{n i}, i=1,2, \cdots, m$, only. In solving this system of equations, one may neglect terms involving $R_{n i}^{2}$ or higher powers.

The direct power series substitution technique together with estimates of error presented above are quite simple to apply in solving differential equations. Moreover, the error estimate for $R_{n i}(t)$ in either of the estimating schemes has the property that

$$
\left|R_{n i}(t)\right| \leq \max \left|\frac{(\Delta t)^{n+1}}{(n+1) !} x^{(n+1)}\left(\eta_{i}\right)\right|
$$

the right-hand side being the usual upper-bound expression in Taylor series.

Space Sciences Division Computer Applications Inc.

Silver Spring, Maryland

1. R. C. Buck, Advanced Calculus, McGraw-Hill, New York, 1956. MR 19, 732.

2. E. L. Ince, Ordinary Differential Equations, Dover, New York, 1944, 1956. MR 6, 65. 